\title{
A dynamical holographic QCD model for chiral symmetry breaking and linear confinement
}

\author{
Danning $\mathrm{Li}^{1}$, Mei Huang ${ }^{1,2}$, and Qi-Shu Yan ${ }^{3}$ \\ 1 Institute of High Energy Physics, Chinese Academy of Sciences, Beijing, China \\ 2 Theoretical Physics Center for Science Facilities, \\ Chinese Academy of Sciences, Beijing, China and \\ 3 College of Physical Sciences, Graduate University of Chinese Academy of Sciences, Beijing, China
}

(Dated: October 18, 2018)

\begin{abstract}
A self-consistent holographic QCD model is proposed which can realize both chiral symmetry breaking and confinement, two most important phenomena of QCD. It is pointed out that the model can accommodate both Regge spectra of hadrons and the linear potential between quarks. The model is formulated in the framework of graviton-dilaton-scalar system, where the dilaton field is dual to the dimension-2 gluon condensate and can lead to the linear confinement, while the scalar field corresponds to the quark anti-quark condensate and can explain the property of chiral dynamics.
\end{abstract}

PACS numbers:

Quantum Chromodynamics (QCD) has been accepted as the basic theory of describing strong interaction for more than 30 years. However, it is still a challenge to solve QCD in non-perturbative region where gauge interaction is strong. The discovery of the anti-de Sitter/conformal field theory (AdS/CFT) correspondence and the conjecture of the gravity/gauge duality [1] provides a revolutionary method to tackle the problem of strongly coupled gauge theories. A string description of realistic QCD has not been successfully formulated yet. Many efforts have been invested in searching for such a realistic description by using the "bottom-up" approach. Recent reviews on 5D holographic QCD models can be found in [2].

It is well-known that the QCD vacuum is characterized by spontaneous chiral symmetry breaking and color charge confinement. The spontaneous chiral symmetry breaking is well understood by the dimension-3 quark condensate $\langle\bar{q} q\rangle[3]$ in the vacuum, in spite of that, the understanding to confinement remains a challenge. Confinement can be reflected by the Regge trajectories of hadrons [4], which suggests that the color charge can form the string-like structure inside hadrons. It can also be shown by the linear potential between two quarks (either light or heavy) at large distances, i.e. $V_{\bar{Q} Q}(R)=\sigma_{s} R$ with $\sigma_{s}$ the string tension.

A successful holographic QCD model should describe both the Regge trajectories of hadron spectra and linear quark potential. Nonetheless, the models on market can not accommodate both.

Currently a working framework used to describe the Regge trajectories of hadron spectra is the soft-wall $\mathrm{AdS}_{5}$ model or Karch-Katz-Son-Stephanov (KKSS) model [5], in which the metric is still $\mathrm{AdS}_{5}$, but a quadratic dilaton background is introduced in the $5 \mathrm{D}$ action. Its extended versions, by including a correction in the $5 \mathrm{D}$ action [6, 7] or a modified AdS/CFT dictionary [8], are widely used to investigate the meson spectra and baryon spectra. However, with $\mathrm{AdS}_{5}$ metric, only Coulomb potential between the two quarks can be produced [9].

The working holographic QCD model to realize the linear quark potential was proposed by Andreev and Zakharov, who introduced a positive quadratic correction in the deformed warp factor of $\mathrm{AdS}_{5}$ geometry [10]. (The linear heavy quark potential can also be obtained by introducing other deformed warp factors as in Refs. [11, 12].) The positive quadratic correction in the deformed warp factor in some sense behaves as a negative dilaton background in the $5 \mathrm{D}$ action, which motivates the proposal of the negative dilaton soft-wall model [13, 14]. More discussions on the sign of the dilaton correction can be found in [15, 16].

It is observed that a quadratic background correction in the metric and dilaton background can lead to the linear quark anti-quark potential and the linear Regge behavior, respectively. It is interesting to explore how to generate both the linear Regge behavior of hadron spectra and linear quark potential in a self-consistent model. In this work, a holographic QCD model is proposed and formulated in the graviton-dilaton-scalar coupled system.

Let's first briefly describe the graviton-dilaton coupled system, where the dilaton background is expected to be dual to the effective degree freedom in the pure gluon system. In general, a dilaton background will deform the warp factor of the metric structure. As described in Ref. [17], the metric structure of the holographic QCD model and its corresponding dilaton background can be self-consistently solved from the Einstein equation in the graviton-dilaton coupling system. It is observed in Ref.[18], that the positive quadratic correction in the metric background can describe the pure gluon QCD system quite well. The 5D action of the gravitondilaton system is defined as

$$
S_{G D}=\frac{1}{16 \pi G_{5}} \int d^{5} x \sqrt{g_{s}} e^{-2 \phi}\left(R+4 \partial_{m} \phi \partial^{m} \phi-V_{\phi}\right) .
$$

Where $G_{5}$ is the $5 \mathrm{D}$ Newton constant, $g_{s}, \phi$ and $V_{\phi}$ are the $5 \mathrm{D}$ metric, the dilaton field and dilaton potential in 
the string frame, respectively. Under the quadratic dilaton background

$$
\phi=\mu^{2} z^{2},
$$

the analytic solution of the dilaton potential in the Einstein frame $V_{\Phi}^{E}=e^{4 \phi / 3} V_{\phi}$ with $\phi=\sqrt{\frac{3}{8}} \Phi$ takes the form of

$$
V_{\Phi}^{E}=-12 \frac{{ }_{0} F_{1}\left(1 / 4 ; \frac{\Phi^{2}}{24}\right)^{2}}{L^{2}}+2 \frac{{ }_{0} F_{1}\left(5 / 4 ; \frac{\Phi^{2}}{24}\right)^{2} \Phi^{2}}{L^{2}}
$$

here $L$ the radius of $\mathrm{AdS}_{5}$ and ${ }_{0} F_{1}(a ; z)$ the hypergeometric function. In the ultraviolet limit, $V_{\Phi}^{E} \stackrel{\Phi \rightarrow 0}{\longrightarrow}$ $-\frac{12}{L^{2}}-\frac{1}{2} M_{\Phi}^{2} \Phi^{2}$ with the 5D mass for the dilaton field $M_{\Phi}^{2} L^{2}=4$. From the AdS/CFT dictionary $\Delta(4-\Delta)=$ $M_{\Phi}^{2} L^{2}$, one can derive its dimension $\Delta=2$. The most likely dimension- 2 operator candidate is $A_{\mu}^{2}$. It has been pointed out in Refs. 19, 20] that the dimension-2 gluon condensate plays essential role for the linear confinement. Therefore, we can regard the quadratic dilaton field is dual to the dimension-2 gluon condensate, i.e., $<A_{\mu}^{2}>=\mu^{2}$, and the graviton-dilaton system describes the pure gluodynamics.

We now add the probe of flavor dynamics on the pure gluodynamic background, and extend the gravitondilaton system to the framework of graviton-dilatonscalar coupling system, where the scalar field captures chiral dynamics. The graviton-dilaton-scalar system can be described by the following $5 \mathrm{D}$ action:

$$
S=S_{G D}+S_{M},
$$

with $S_{G D}$ given in Eq.(1) and $S_{M}$ the KKSS action for mesons as in [5] taking the form of

$$
\begin{aligned}
S_{M}= & -\frac{N_{f}}{N_{c}} \int d^{5} x \sqrt{g_{s}} e^{-\phi} \operatorname{Tr}\left(|D X|^{2}+V_{X}\right. \\
& \left.+\frac{1}{4 g_{5}^{2}}\left(F_{L}^{2}+F_{R}^{2}\right)\right) .
\end{aligned}
$$

Where $X$ and $V_{X}$ are the scalar field and its corresponding potential. $g_{5}^{2}$ is fixed as $12 \pi^{2} N_{f} / N_{c}^{2}$ [5] and we take $N_{f}=2, N_{c}=3$ in this paper.

We assume the vacuum background is induced by the dilaton field of dimension- 2 gluon condensate and the scalar field of the quark antiquark condensate $\langle X\rangle=$ $\frac{\chi(z)}{2}[5]$, then the vacuum background part of the action Eq.(44) takes the following form

$$
\begin{aligned}
S_{v a c} & =\frac{1}{16 \pi G_{5}} \int d^{5} x \sqrt{g_{s}}\left\{e^{-2 \phi}\left(R_{s}+4 \partial_{m} \phi \partial^{m} \phi-V_{\phi}\right)\right. \\
& \left.-\lambda e^{-\phi}\left(\frac{1}{2} \partial_{m} \chi \partial^{m} \chi+V_{\chi}\right)\right\}
\end{aligned}
$$

with $\lambda=\frac{16 \pi G_{5} N_{f}}{N_{c} L^{3}}$ and the metric in the string frame

$$
d S_{s}^{2}=B_{s}^{2}\left(-d t^{2}+d \vec{x}^{2}+d z^{2}\right)
$$

where

$$
B_{s}^{2} \equiv e^{2 A_{s}} \equiv L^{2} b_{s}^{2} .
$$

It is easy to derive the following three coupled field equations:

$$
\begin{aligned}
-A_{s}^{\prime \prime}+A_{s}^{\prime 2}+\frac{2}{3} \phi^{\prime \prime}-\frac{4}{3} A_{s}^{\prime} \phi^{\prime}-\frac{\lambda}{6} e^{\phi} \chi^{\prime 2} & =0, \\
\phi^{\prime \prime}+\left(3 A_{s}^{\prime}-2 \phi^{\prime}\right) \phi^{\prime}-\frac{3 \lambda}{16} e^{\phi} \chi^{\prime 2} & \\
-\frac{3}{8} e^{2 A_{s}-\frac{4}{3} \phi} \partial_{\phi}\left(e^{4 / 3 \phi} V_{\phi}+\lambda e^{7 / 3 \phi} V_{\chi}\right) & =0, \\
\chi^{\prime \prime}+\left(3 A_{s}^{\prime}-\phi^{\prime}\right) \chi^{\prime}-e^{2 A_{s}} \partial_{\chi} V_{\chi} & =0 .
\end{aligned}
$$

If we know the dynamical information of the dilaton field $\phi$ and the scalar field $\chi$, then the metric $A_{s}$, the dilaton potential $V_{\phi}$ and the scalar potential $V_{\chi}$ should be selfconsistently solved from the above three coupled equations. It is noticed that the graviton-dilaton-scalar system is different from the graviton-dilaton-tachyon system [21], where the metric remains as $\mathrm{AdS}_{5}$.

The UV asymptotic form of $\chi(z)$. - As proposed in the KKSS model, at ultraviolet(UV), the scalar field takes the following asymptotic form,

$$
\chi(z) \stackrel{z \rightarrow 0}{\longrightarrow} m_{q} \zeta z+\frac{\sigma}{\zeta} z^{3},
$$

where $m_{q}$ is the current quark mass, and $\sigma$ is the quark antiquark condensate, and $\zeta$ is a normalization constant and is fixed as $\zeta^{2}=\frac{N_{c}^{2}}{4 \pi^{2} N_{f}}$. In this paper, we would fix $m_{q}=5 \mathrm{MeV}, \sigma=(228 \mathrm{MeV})^{3}$.

The IR asymptotic form of $\chi(z)$ constrained from linear quark potential. - The linear behavior of quarkantiquark static potential in the heavy quark mass limit $m_{Q} \rightarrow \infty$ can describe the permanent confinement property of QCD. Following Ref. [9], one can solve the renormalized free energy of the $\bar{q} q$ system under the general metric background $A_{s}$,

$$
\begin{aligned}
& V_{\bar{q} q}\left(z_{0}\right)=\frac{g_{p}}{\pi z_{0}}\left(\int_{0}^{1} d \nu\left(\frac{b_{s}^{2}\left(z_{0} \nu\right) z_{0}^{2}}{\sqrt{1-\frac{b_{s}^{4}\left(z_{0}\right)}{b_{s}^{4}\left(z_{0} \nu\right)}}}-\frac{1}{\nu^{2}}\right)-1\right), \\
& R_{\bar{q} q}\left(z_{0}\right)=2 z_{0} \int_{0}^{1} d \nu \frac{1}{\sqrt{1-\frac{b_{s}^{4}\left(z_{0}\right)}{b_{s}^{4}\left(z_{0} \nu\right)}}} \frac{b_{s}^{2}\left(z_{0}\right)}{b_{s}^{2}\left(z_{0} \nu\right)} .
\end{aligned}
$$

Here $g_{p}=\frac{L^{2}}{\alpha}$ and $\alpha$ the $5 \mathrm{D}$ effective string tension. The integrate kernel in Eq. (13) has a pole at $\nu=1$, expanding the integral kernel at $\nu=1$ one has

$$
1-\frac{b_{s}^{4}\left(z_{0}\right)}{b_{s}^{4}\left(z_{0} \nu\right)}=\frac{4 z_{0} b_{s}^{\prime}\left(z_{0}\right)}{b_{s}\left(z_{0}\right)}(\nu-1)+o\left((\nu-1)^{2}\right)
$$

From Eqs.13[14]15), one can observe that at the point $z_{c}$ when $b_{s}^{\prime}\left(z_{c}\right) \rightarrow 0$, then the integral is dominated by $\nu=1$ region, one can have

$$
\frac{V_{\bar{q} q}\left(z_{0}\right)}{R_{\bar{q} q}\left(z_{0}\right)} \stackrel{z_{0} \rightarrow z_{c}}{\longrightarrow} \frac{g_{p}}{2 \pi} b_{s}^{2}\left(z_{c}\right) .
$$


Therefore, the necessary condition for the linear part of the $q-\bar{q}$ potential is that there exists one point $z_{c}$ or one region, where $b_{s}^{\prime}(z) \rightarrow 0, z \rightarrow z_{c}$ while $b_{s}(z)$ keeps finite. For simplicity, we can take the following constraint on the metric structure at IR:

$$
A_{s}^{\prime}(z) \stackrel{z \rightarrow \infty}{\longrightarrow} 0, A_{s}(z) \stackrel{z \rightarrow \infty}{\longrightarrow} \text { Const. }
$$

Under the condition of Eq.(17), the equation of motion Eq.(9) in the IR takes the following simple form:

$$
\frac{2}{3} \phi^{\prime \prime}-\frac{\lambda}{6} e^{\phi} \chi^{\prime 2}=0,
$$

which provides a relation between the chiral condensate and dimension-2 gluon condensate at IR. The asymptotic form of $\chi(z)$ at IR can be solved as:

$$
\chi(z) \stackrel{z \rightarrow \infty}{\longrightarrow} \sqrt{8 / \lambda} \mu e^{-\phi / 2} .
$$

To match the asymptotic forms both at UV and IR in Eqs.(12) and (19), $\chi$ can be parameterized as

$$
\chi^{\prime}(z)=\sqrt{8 / \lambda} \mu e^{-\phi / 2}\left(1+c_{1} e^{-\phi}+c_{2} e^{-2 \phi}\right)
$$

with $c_{1}=-2+\frac{5 \sqrt{2 \lambda} m_{q} \zeta}{8 \mu}+\frac{3 \sqrt{2 \lambda} \sigma}{4 \zeta \mu^{3}}$ and $c_{2}=1-\frac{3 \sqrt{2 \lambda} m_{q} \zeta}{8 \mu}-$ $\frac{3 \sqrt{2 \lambda} \sigma}{4 \zeta \mu^{3}}$.

Regge trajectories of mesons. - Under the dilaton background Eq.(2) and the scalar profile Eq.(20), the metric structure $A_{s}(z)$ or $b_{s}(z)$ can be solved through Eq. (9). Considering the meson fluctuations under the vacuum background described by $A_{s}, \phi, \chi$ as in [5], we can split the fields into background part and perturbation part. For $X$ we would have a scalar perturbation $s$ and pseudo-scalar perturbation $\pi$, i.e., $X=\left(\frac{\chi}{2}+s\right) e^{12 \pi^{a} t^{a}}$. For the vector field $V_{\mu}$ and axial vector field $A_{\mu}$ part, due to their vanishing vacuum expectation value, we would use the same notation to denote the perturbation fields. The equations of motion for perturbation fields $s, \pi, V_{\mu}, A_{\mu}$ can be easily derived. For example, the schrodinger like equation for vector is given below:

$$
\begin{aligned}
& -v_{n}^{\prime \prime}+V_{v}(z) v_{n}=m_{n, v}^{2} v_{n}, \\
& V_{v}(z)=\frac{A_{s}^{\prime \prime}-\phi^{\prime \prime}}{2}+\frac{\left(A_{s}^{\prime}-\phi^{\prime}\right)^{2}}{4} .
\end{aligned}
$$

It is noticed that at IR, $V_{v}(z)=-\mu^{2}+\mu^{4} z^{2}$, therefore the solution of Eq.(22) for high excitations is $M_{n}^{2}=4 \mu^{2} n$.

By fixing the 5D Newton constant $G_{5}=\frac{3 L^{3}}{4}$, one can produce the proper splitting between the vector and axial vector Regge trajectories. The produced spectra of scalar $f_{0}$, pseudoscalar $\pi$, vector $\rho$ and axialvector $a_{1}$ are shown in Fig 1 compared with experimental data [22]. It is worth of mentioning that the experimental data for $f_{0}$ are chosen as in Ref. [6]. With only 4 parameters, all produced meson spectra in the graviton-dilaton-scalar system agree well with experimental data except $f_{0}(600)$. It is observed that the Regge trajectories are parallel and the slope is $4 \mu^{2}$ with $\mu=0.43 \mathrm{GeV}$.

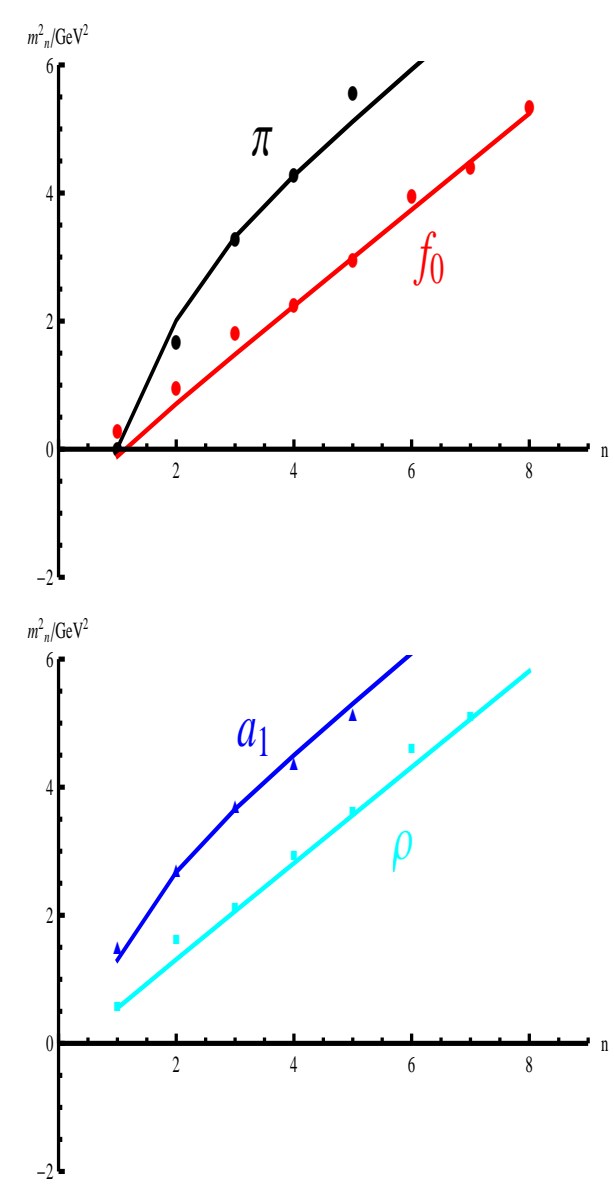

FIG. 1: A plot of experimental(dot) and model predicted (line) mass square spectra for the scalar and pseudoscalar mesons $f_{0}, \pi$ and vector and axial-vector mesons $\rho, a_{1}$.

The string tension of the linear quark potential. - Under the metric background $A_{s}(z)$ solved from Eq.(9) with the dilaton background Eq.(2) and the scalar profile Eq.(20), the quark potential can be solved from Eqs.(13) and (14). In the UV limit, one can derive the Coulomb potential $V_{\bar{q} q}=-\frac{0.23 g_{p}}{R_{\bar{q} q}}$ as given in Ref.[9]. In the IR limit, we can get the linear potential $V_{\bar{q} q}=\frac{g_{p}}{2 \pi} b_{s}^{2}\left(z_{c}\right) R_{\bar{q} q}$. From the solutions in Eq.(9), we have $b_{s}^{2} \approx 4 \mu^{2}$, which indicates that the string tension of the linear quark potential $\sigma_{s} \sim 4 \mu^{2}$.

The numerical result for the quark potential $V_{\bar{q} q}$ as a function of quark anti-quark distance $R_{q q}$ is shown by the solid line in Fig 2. The two parameters in Eq.(13) are fixed as $g_{p}=2.3$ and $\mu=0.43 \mathrm{GeV}$. The result agrees with the Cornell potential (dot-dashed line) [23] $V^{c}(R)=$ $-\frac{\kappa}{R}+\sigma_{s t r} R+V_{0}$ with $\kappa \approx 0.48, \sigma_{\text {str }} \approx 0.183 \mathrm{GeV}^{2}$ and $V_{0}=-0.25 \mathrm{GeV}$.

The last but not the least, we discuss the sign of the dilaton background. If we choose a negative dilaton background $\phi=-\mu^{2} z^{2}$ as in Ref.[13, 14], in the IR limit, the last term in Eq.(9) decreases exponentially to zero, 


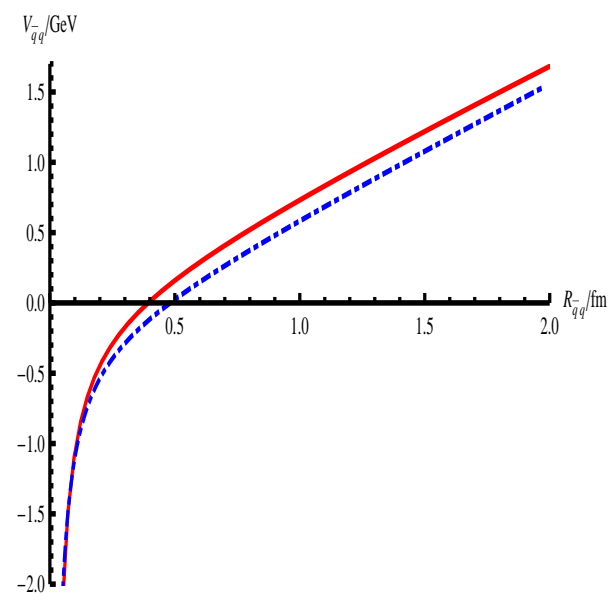

FIG. 2: $V_{\bar{q} q}$ as a function of $R_{\bar{q} q}$ from our model (solid line) with $g_{p}=2.3$ and $\mu=0.43 \mathrm{GeV}$ compared with the Cornell potential (dot-dashed line).

and one can get the asymptotic solution of $A_{s}(z) \stackrel{z \rightarrow \infty}{\longrightarrow}$ $-\frac{4}{3} \mu^{2} z^{2}+1 / 2 \log (z)$ and $b_{s}(z) \sim \sqrt{z} e^{-\frac{4}{3} \mu^{2} z^{2}} \stackrel{z \rightarrow \infty}{\longrightarrow} 0$. From Eq.(16), it's not possible to produce the linear potential with a negative dilaton background. Therefore, one can safely exclude the negative dilaton background in the graviton-dilaton-scalar system.
In summary, we propose a dynamical holographic QCD model, which takes into account the back-reaction of flavor dynamics on the pure gluodynamic background. To our knowledge, this is the first dynamical holographic QCD model which can produce both the linear Regge trajectories of hadron spectra and quark anti-quark linear potential. It is observed that both the slope of the Regge trajectories and the string tension of the linear quark anti-quark potential are proportional to the dimension-2 gluon condensate. This result indicates that the linear confinement is dynamically induced by the dimension- 2 gluon condensate. The holographic QCD model offers us a new viewpoint on the relation between the chiral symmetry breaking and confinement. It is found that the balance between the chiral condensate and dimension2 gluon condensate is essential to produce the correct Regge behavior of hadron spectra. As a byproduct, it is found that the negative dilaton background can be safely excluded in the framework of graviton-dilaton-scalar system.

Acknowledgement.- We thank valuable discussions with S. He, C. Liu, Y.X. Liu, J.J. Wu, J.B. Wu and F.K. $\mathrm{Xu}$. This work is supported by the NSFC under Grant No. 11175251, CAS fellowship for young foreign scientists under Grant No. 2011Y2JB05, CAS key project KJCX2EW-N01, K.C.Wong Education Foundation, and Youth Innovation Promotion Association of CAS.
[1] J. M. Maldacena, Adv. Theor. Math. Phys. 2, 231 (1998) [Int. J. Theor. Phys. 38, 1113 (1999)]; S. S. Gubser, I. R. Klebanov and A. M. Polyakov, Phys. Lett. B 428, 105 (1998); E. Witten, Adv.Theor.Math.Phys. 2 (1998) 253-291.

[2] G. F. de Teramond and S. J. Brodsky, arXiv:1203.4025 [hep-ph]. Y. Kim, I. J. Shin and T. Tsukioka, arXiv:1205.4852 [hep-ph]. A. Adams, L. D. Carr, T. Schaefer, P. Steinberg and J. E. Thomas, arXiv:1205.5180 [hep-th].

[3] Y. Nambu, Phys. Rev. 117, 648-663 (1960).

[4] G. Veneziano, Nuovo Cim. A 57, 190 (1968); P.D.B. Collins, An Introduction to Regge Theory and High Energy Physics, Cambridge Univ. Press, Cambridge (1975).

[5] A. Karch, E. Katz, D. T. Son and M. A. Stephanov, Phys. Rev. D 74 (2006) 015005

[6] T. Gherghetta, J. I. Kapusta and T. M. Kelley, Phys. Rev. D 79 (2009) 076003; T. M. Kelley, S. P. Bartz and J. I. Kapusta, Phys. Rev. D 83 (2011) 016002; T. M. Kelley, arXiv:1108.0653 [hep-ph].

[7] Y. -Q. Sui, Y. -L. Wu, Z. -F. Xie and Y. -B. Yang, Phys. Rev. D 81 (2010) 014024; Y. -Q. Sui, Y. -L. Wu and Y. -B. Yang, Phys. Rev. D 83 (2011) 065030.

[8] S. J. Brodsky and G. F. de Teramond, Phys. Lett. B 582, 211 (2004); T. Branz, T. Gutsche, V. E. Lyubovitskij, I. Schmidt and A. Vega, Phys. Rev. D 82, 074022 (2010)

[9] J. M. Maldacena, Phys. Rev. Lett. 80 (1998) 4859

[10] O. Andreev and V. I. Zakharov, Phys. Rev. D 74, 025023
(2006).

[11] H. J. Pirner and B. Galow, Phys. Lett. B 679, 51 (2009)

[12] S. He, M. Huang and Q. S. Yan, Phys. Rev. D 83, 045034 (2011).

[13] F. Zuo, Phys. Rev. D 82, 086011 (2010).

[14] G. F. de Teramond and S. J. Brodsky, Baryon Spectra," arXiv:0909.3900 [hep-ph].

[15] T. Gutsche, V. E. Lyubovitskij, I. Schmidt and A. Vega, Phys. Rev. D 85, 076003 (2012).

[16] A. Karch, E. Katz, D. T. Son and M. A. Stephanov, JHEP 1104, 066 (2011).

[17] U. Gursoy and E. Kiritsis, JHEP 0802 (2008) 032; U. Gursoy, E. Kiritsis and F. Nitti, JHEP 0802 (2008) 019 .

[18] D. Li, S. He, M. Huang and Q. -S. Yan, JHEP 1109 (2011) 041.

[19] F. V. Gubarev, L. Stodolsky, V. I. Zakharov, Phys. Rev. Lett. 86, 2220-2222 (2001); K. I. Kondo, Phys. Lett. B 514, 335 (2001).

[20] F. Xu and M. Huang, arXiv:1111.5152 [hep-ph].

[21] B. Batell and T. Gherghetta, Phys. Rev. D 78 (2008) 026002; J. I. Kapusta and T. Springer, Phys. Rev. D 81, 086009 (2010); T. M. Kelley, arXiv:1107.0931 [hep-ph].

[22] C. Amsler et al. [Particle Data Group Collaboration], Phys. Lett. B 667 (2008) 1.

[23] E. Eichten, K. Gottfried, T. Kinoshita, K. D. Lane and T. M. Yan, Phys. Rev. D 21, 203 (1980). 